\title{
Subcortical Atrophy Is Associated with Cognitive Impairment in Mild Parkinson Disease: A Combined Investigation of Volumetric Changes, Cortical Thickness, and Vertex-Based Shape Analysis
}

\author{
E. Mak, N. Bergsland, M.G. Dwyer, R. Zivadinov, and N. Kandiah
}

\begin{abstract}
BACKGROUND AND PURPOSE: The involvement of subcortical deep gray matter and cortical thinning associated with mild Parkinson disease remains poorly understood. We assessed cortical thickness and subcortical volumes in patients with Parkinson disease without dementia and evaluated their associations with cognitive dysfunction.
\end{abstract}

MATERIALS AND METHODS: The study included 90 patients with mild Parkinson disease without dementia. Neuropsychological assessments classified the sample into patients with mild cognitive impairment $(n=25)$ and patients without cognitive impairment $(n=65)$. Volumetric data for subcortical structures were obtained by using the FMRIB Integrated Registration and Segmentation Tool while whole-brain, gray and white matter volumes were estimated by using Structural Image Evaluation, with Normalization of Atrophy. Vertex-based shape analyses were performed to investigate shape differences in subcortical structures. Vertex-wise group differences in cortical thickness were also assessed. Volumetric comparisons between Parkinson disease with mild cognitive impairment and Parkinson disease with no cognitive impairment were performed by using ANCOVA. Associations of subcortical structures with both cognitive function and disease severity were assessed by using linear regression models.

RESULTS: Compared with Parkinson disease with no cognitive impairment, Parkinson disease with mild cognitive impairment demonstrated reduced volumes of the thalamus $(P=.03)$ and the nucleus accumbens $(P=.04)$. Significant associations were found for the nucleus accumbens and putamen with performances on the attention/working memory domains $(P<.05)$ and nucleus accumbens and language domains $(P=.04)$. The 2 groups did not differ in measures of subcortical shape or in cortical thickness.

CONCLUSIONS: Patients with Parkinson disease with mild cognitive impairment demonstrated reduced subcortical volumes, which were associated with cognitive deficits. The thalamus, nucleus accumbens, and putamen may serve as potential biomarkers for Parkinson disease-mild cognitive impairment.

ABBREVIATIONS: $\mathrm{MCI}=$ mild cognitive impairment; $\mathrm{MDS}=$ Movement Disorder Society; $\mathrm{PD}=$ Parkinson disease; $\mathrm{PD}-\mathrm{MCl}=$ Parkinson disease with mild cognitive impairment; $\mathrm{PD}-\mathrm{NCI}$ = Parkinson disease with no cognitive impairment; $\mathrm{SDGM}=$ subcortical deep gray matter; SIENAX = Structural Image Evaluation, with Normalization of Atrophy

P arkinson disease (PD) has traditionally been considered a motor disorder. However, the presence of cognitive dysfunction is increasingly recognized and known to occur even at early stages,

\section{Received November 8, 2013; accepted after revision May 28, 2014.}

From the Department of Neurology (E.M., N.B., M.G.D., R.Z.), Buffalo Neuroimaging Analysis Center, and MR Imaging Clinical Translational Research Center (R.Z.), School of Medicine and Biomedical Sciences, University at Buffalo, State University of New York, Buffalo, New York; Department of Neurology (E.M., N.K.), National Neuroscience Institute, Singapore; and Istituto de Ricovero e Cura a Carattere Scientifico "S. Maria Nascente," Don Gnocchi Foundation (N.B.), Milan, Italy.

This work was supported by the Singapore National Research Foundation and Singapore Ministry of Health's National Medical Research Council and by the Buffalo Neuroimaging Analysis Center neuroimaging fellowship to Elijah Mak.

Please address correspondence to Robert Zivadinov, MD, PhD, FAAN, Department of Neurology, School of Medicine and Biomedical Sciences, Jacobs Neurological Institute, 100 High St, Buffalo, NY 14203; e-mail: rzivadinov@bnac.net; or Nagaendran Kandiah, MBBS, MRCP (UK), FAM, Department of Neurology, National Neuroscience Institute, 11 Jalan Tan Tock Seng, Singapore 308433; e-mail: nagaendran kandiah@nni.com.sg and most patients develop dementia during the course of the disease. Recently, it has emerged that patients with PD show a wide and variable spectrum of cognitive deficits involving multiple domains such as executive function, attention, memory, visuospatial, and, less frequently, language. ${ }^{1,2}$ While traditionally believed to occur only in advanced stages of PD, recent studies suggest that approximately $30 \%-35 \%$ of patients with early PD experience cognitive disturbances, ${ }^{3,4}$ which have been defined as mild cognitive impairment (MCI). ${ }^{5}$ The Movement Disorder Society (MDS) Task Force reported a mean prevalence of Parkinson disease with mild cognitive impairment (PD-MCI) at 27\%, ranging from $19 \%$ to $38 \%{ }^{6}$ Furthermore, the impact of MCI and dementia in pa-

\footnotetext{
- Indicates open access to non-subscribers at www.ajnr.org

$\equiv$ Indicates article with supplemental on-line table.

http://dx.doi.org/10.3174/ajnr.A4055
} 
tients with PD at any given stage of the disease is substantial, with adverse consequences for functioning, ${ }^{7}$ psychiatric morbidity, caregiver burden, ${ }^{8}$ and mortality. ${ }^{9}$ At present, there is much to be elucidated with regard to the etiology of cognitive impairment in PD.

Initially, dementia in PD was described as subcortical. Cognitive dysfunction in patients without dementia has also been attributed to dopaminergic depletion disrupting the frontostriatal circuit $^{10}$ or dopamine-acetylcholine synaptic imbalance. ${ }^{11} \mathrm{Nev}-$ ertheless, recent investigations by using structural MR imaging suggest that specific cognitive deficits, such as memory deficits, and dementia in PD may also be accompanied by structural cerebral abnormalities. In this regard, MR imaging studies have demonstrated cortical atrophy in patients with $\mathrm{PD}$ with dementia. A recent meta-analysis revealed regional gray matter reductions of the medial temporal lobe and the basal ganglia, ${ }^{12}$ while other areas, including the caudate, ${ }^{13}$ hippocampus, ${ }^{14}$ and amygdala, ${ }^{15}$ have also been implicated. However, present findings on GM atrophy in patients without dementia with $\mathrm{PD}$ are inconclusive. While a few studies have demonstrated atrophy in the medial temporal lobes, ${ }^{16}$ amygdala, ${ }^{17}$ and frontal and parietal regions, ${ }^{18}$ others have reported no significant GM reductions in PD populations without dementia. ${ }^{19}$

In addition, cortical thinning in PD represents a relatively new area of research, and it has been reported to be more sensitive than voxel-based morphometry. ${ }^{20}$ Recent studies have shown that cortical thinning occurs in PD without dementia. ${ }^{21}$ A longitudinal study also reported that patients with early PD presented with a more aggressive rate of cortical thinning in the frontotemporal regions compared with healthy controls. ${ }^{22}$

These mixed neuroimaging findings could be due, in part, to cognitively heterogeneous groups of patients, particularly in studies in which patients with MCI were not distinguished from those with normal cognition. Therefore, to systematically compare the pattern of GM atrophy in mild PD and its impact on specific cognitive domains, we used the recent MDS Task Force criteria to classify patients with PD with MCI or as cognitively normal (PDNCI). We estimated the volumes of the amygdala, hippocampus, nucleus accumbens, caudate nucleus, putamen, pallidum, and thalamus in a cohort of patients with PD by using the FMRIB Integrated Registration and Segmentation Tool (FIRST; http:// fsl.fmrib.ox.ac.uk/fsl/fslwiki/FIRST).

Furthermore, we assessed differences in subcortical deep gray matter (SDGM) structures between PD-MCI and PD-NCI and further examined associations between individual structures and cognitive performances across multiple domains. Because vertex analysis directly measures changes in geometry without any smoothing of the image data, it might have the potential to more precisely detect regional alterations of the subcortical GM than the conventional voxel-based morphometry approach. ${ }^{23}$ Therefore, we used a vertex-based shape-analysis method to investigate potential shape differences of SDGM structures between PD-MCI and PD-NCI. Last, vertex-wise cortical thickness analysis was performed by using FreeSurfer (http://surfer.nmr.mgh.harvard.edu) to assess and compare patterns of regional cortical alterations between both PD groups.

\section{MATERIALS AND METHODS \\ Subjects}

The present study included 90 patients with mild PD (65.08 \pm 7.71 years of age; disease duration, $5.26 \pm 3.90$ years; and Hoehn and Yahr stage $=1.88 \pm 0.39$ ) recruited from August 2011 to March 2012 from a tertiary neurology center. PD was diagnosed by neurologists trained in movement disorders according to the National Institute of Neurologic Disorders and Stroke criteria. ${ }^{24}$ Patients with dementia and serious medical and psychiatric comorbidities were excluded.

\section{Clinical Assessment}

Demographic, clinical, and vascular risk factor data were collected, and a comprehensive clinical assessment was conducted to ascertain cognitive status and functional ability. The severity and stage of the patient's parkinsonism was evaluated by using the Unified Parkinson's Disease Rating Scale motor subscore ${ }^{25}$ and the modified Hoehn and Yahr stage. ${ }^{26}$ To standardize data on medication use, we converted dosages of PD medications to total daily levodopa-equivalent doses. This calculation was based on the conversion formulae reported by Tomlinson et al. ${ }^{27}$ The study was approved by the centralized institutional review boards of the participating institutions, and informed consent was obtained from patients or their legal caregivers.

\section{Neuropsychological Assessment}

Cognitive performance was evaluated by trained psychologists by using a standardized neuropsychological battery. To ensure standardization and integrity of data, we evaluated patients during their "on" medication state. Global cognition was evaluated by using the Mini-Mental State Examination ${ }^{28}$ and the Montreal Cognitive Assessment. ${ }^{29}$ In line with the recommendations of the MDS Task Force, specific cognitive domains including memory, executive function, visuospatial function and language, and attention/working memory were also assessed. ${ }^{30}$ In this regard, patients were evaluated with the following subtests from the Alzheimer Disease Assessment Scale-Cognitive ${ }^{31}$ : Word-List Immediate, Delayed and Recognition Recall for episodic memory; the Frontal Assessment Battery and the 10-Point Clock Test for executive function; a figure copy test and test for the number of errors made on a maze for visuospatial function; a 20-point object-naming test and a fruit-naming fluency test for language assessment; and digit span, color trails 2 , and a test for time taken on a maze for attention/working memory. ${ }^{31-33}$ Performances on individual tasks were transformed into $z$ scores. Subsequently, a composite summary index for each cognitive domain was derived from the corresponding averages of the respective individual neuropsychological tests.

\section{Classification}

To qualify PD-MCI for MDS level 2 criteria, we analyzed performance on the suggested 5 cognitive domains (attention/working memory, executive, language, episodic memory, and visuospatial). Cutoff scores for the various cognitive tests were based on locally validated norms when available, and for those without, international ones were used. The performance on a cognitive test was considered abnormal if the score was 1.5 SDs below the norm. 
Impairment on at least 2 neuropsychological tests, represented by either 2 tests showing impairment in 1 cognitive domain or 1 test showing impairment in 2 different cognitive domains, was required. Patients with PD who did not fulfill the criteria for PDMCI or PD-dementia were classified as PD-NCI.

\section{Image Acquisition}

All subjects underwent MR imaging on a 3T whole-body system (Achieva 3.0; Philips Healthcare, Best, the Netherlands). A highresolution volumetric 3D T1-weighted magnetization-prepared rapid acquisition of gradient echo sequence (axial acquisition: TR, $7.1 \mathrm{~ms}$; TE, $3.3 \mathrm{~ms}$; TI, $850 \mathrm{~ms}$; FOV, $240 \times 240 \mathrm{~mm}^{2}$; matrix, $256 \times 256$; section thickness, $1 \mathrm{~mm}$; total, 180 sections; scanning time, 5 minutes and 13 seconds) and a whole-brain 3D fluidattenuated inversion recovery sequence (turbo spin-echo: TR, $8000 \mathrm{~ms}$; TE, $340 \mathrm{~ms}$; TI, $2400 \mathrm{~ms}$; FOV, $240 \times 240 \mathrm{~mm}^{2}$; matrix, $256 \times 256$; section thickness, $1 \mathrm{~mm}$; total, 170 sections; scanning time, 10:24) were acquired for all patients. Both clinical testing and MR imaging were performed on the same day for all patients.

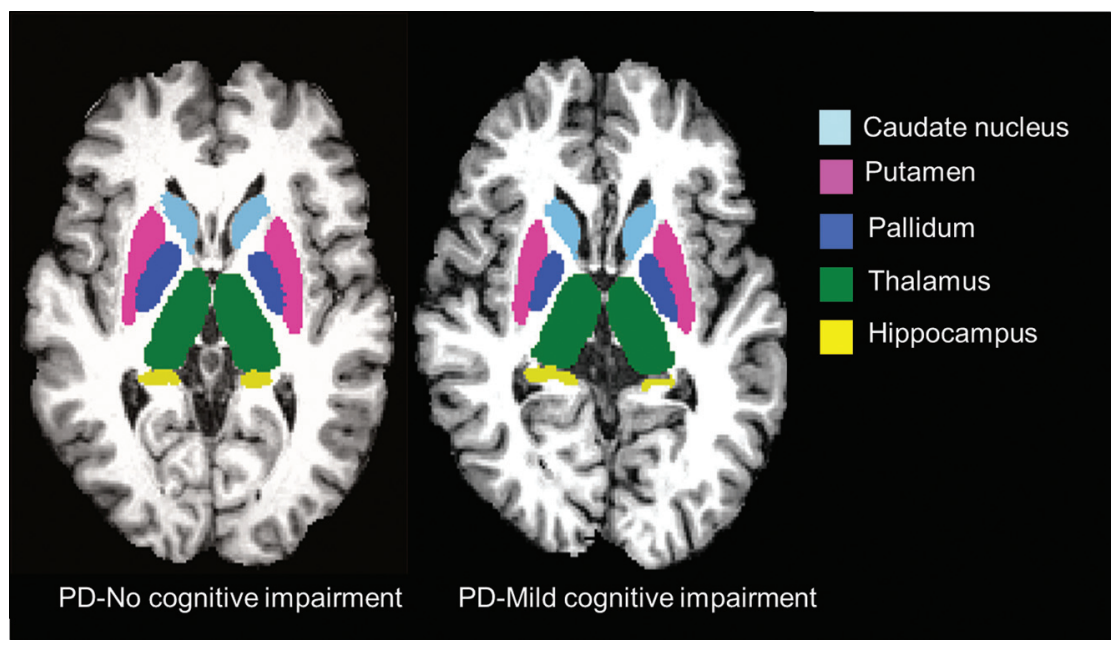

FIG 1. Representative FIRST segmentation of subcortical structures in patients with PD-MCI (left) and PD-NCl (right).

\section{Image Analysis}

Quantitative image analyses were performed at the Buffalo Neuroimaging Analysis Center, Buffalo, New York.

Quantification of GM and WM Volumes. For each subject, we obtained GM, white matter, and a volumetric scaling factor by using Structural Image Evaluation, with Normalization of Atrophy (SIENAX, Version 2.6; FMRIB Software Library, http://www.fmrib.ox.ac.uk/fsl/). ${ }^{34}$

Quantification of Subcortical Deep Gray Matter Volumes. Before we used the T1-weighted images for subsequent analysis, the analysis was modified by an in-house-developed in-painting technique to avoid the impact of WM hyperintensities on GM volume as previously described. ${ }^{35}$ WM hyperintensities were outlined on each axial FLAIR image section by using a reproducible, semiautomated local threshold technique (Jim, Version 5.0; Xinapse Systems, Northamptonshire, UK). All WM hyperintensity masks were created by a single rater (E.M.), blinded to clinical characteristics and tests results, with similar reproducibility as previously reported. ${ }^{36}$

Subsequently, FIRST was used to segment the amygdala, hippocampus, nucleus accumbens, pallidum, caudate nucleus, putamen, and thalamus from the in-painted $\mathrm{T} 1 .^{23}$ The reproducibility of FIRST has been previously reported. ${ }^{37}$ Examples of subcortical segmentation of a subject classified as having PD-MCI and of a subject classified as having PD-NCI are presented in Fig 1. In subsequent volumetric analyses, the normalization factor from SIENAX was included to reduce the effects of interindividual variability due to head size. ${ }^{34}$

Vertex Analysis for Assessment of SDGM Shape Alterations. FIRST creates a surface mesh for each subcortical structure
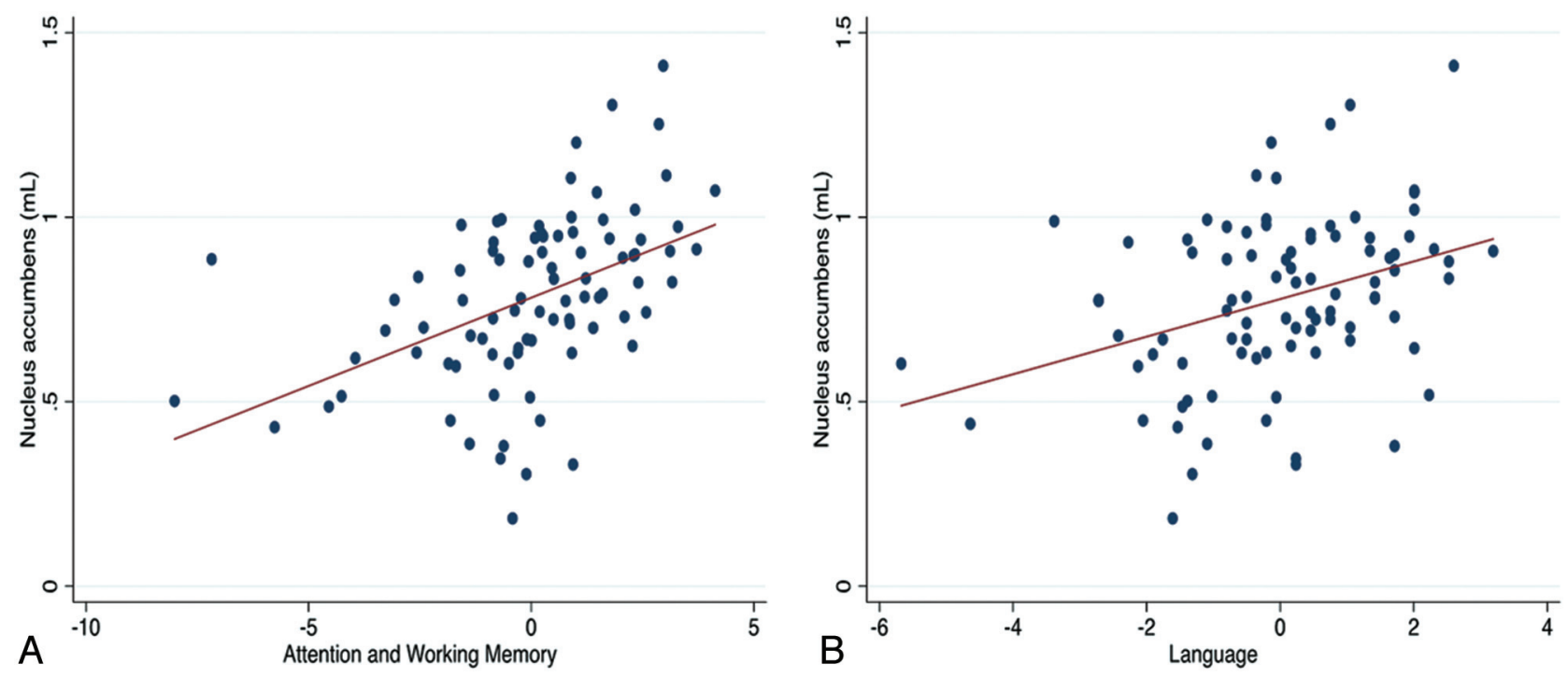

FIG 2. Scatterplot showing the associations between the nucleus accumbens and attention and working memory $(A)$ and language domains $(B)$. 
Table 1: Demographic, clinical, and white matter hyperintensity characteristics between patients with Parkinson disease with and without mild cognitive impairment

Demographic and Clinical Variables

Age (yr) (mean) (SD)

Sex (male) (No.) (\%)

Education (yr) (mean) (SD)

Hoehn and Yahr (mean) (SD)

Disease duration (yr) (mean) (SD)

UPDRS (mean) (SD)

Levodopa equivalent dose (mg) (mean) (SD)

Cardiovascular risk factors (No.) (\%)

Diabetes

Hypertension

Hyperlipidemia

smoking

White matter hyperintensities

Total WMH volume (mm) (mean) (SD)
PD-NCI $(n=65)$

$63.4(7.6)$

46 (72.3)

$11.0(3.1)$

$1.9(0.4)$

5.4 (4.3)

$17.5(7.0)$

$557.4(375.7)$

5 (7.8)

$20(31.3)$

20 (31.3)

$15(23.4)$
$\mathrm{PD}-\mathrm{MCI}(n=25)$

$69.4(6.4)$

18 (76.0)

$9.3(3.5)$

$1.8(0.4)$

$5.0(2.7)$

20.0 (8.4)

$510.2(299.0)$

8 (32.0)

$13(52.0)$

14 (56.0)

6 (24.0)

4.2 (5.8)

Note:-UPDRS indicates Unified Parkinson's Disease Rating Scale, subscore II:

a Significant differences at $P<.05$ level.

${ }^{b}$ Student $t$ test.

${ }^{c} \chi^{2}$ test.

Mann-Whitney $U$ test.

e Analysis of covariance test, corrected for age, sex, and education.

by using a deformable mesh model. The mesh is composed of a set of triangles, and the apex of adjoining triangles is called a vertex. The number of vertices for each structure is fixed so that corresponding vertices can be compared across individuals and between groups. ${ }^{38}$ Vertex analysis was performed, and shape alterations of SDGM were assessed on a per-vertex basis. Regional changes in the vertices of SDGM structures between PD-MCI and PD-NCI were assessed by using a generalized linear model with age, sex, and education as nuisance covariates. The results were then corrected for multiple comparisons by using the false discovery rate $(P<.05)$.

Cortical Thickness Analysis. T1 images of the subjects were processed with the volume and surface pipeline of FreeSurfer. The technical details of cortical reconstruction and volumetric segmentation procedures have been described previously. ${ }^{39}$

\section{Statistical Analysis}

General Analyses. Group comparisons of demographics, neuropsychological variables, and regional volumetric data were performed by using STATA 12 (StataCorp, College Station, Texas). The Student $t$ test or the nonparametric Mann-Whitney rank sum test was used to investigate differences between groups, depending on the normality of the distributions. The $\chi^{2}$ test was used for categoric variables (sex and vascular risk factors). Left and right volumes of the SDGM structures were highly correlated (data not shown). Therefore, to minimize the total number of comparisons, we combined left and right SDGM structures to yield a single structural volume. Mean regional GM volumetric differences between PD-MCI and PD-NCI groups were investigated while controlling for age, sex, education, and head size (by using the volumetric scaling factor from SIENAX) by ANCOVA.

Subsequently, for each SDGM structure showing a significant volumetric difference, a linear regression model was designed to assess associations with different cognitive domains separately. In addition, we also tested for associations between the SDGM structures and disease characteristics (disease duration and Unified Parkinson's Disease Rating Scale motor

$P$ Value

$.001^{\mathrm{a}, \mathrm{b}}$

$.723^{c}$

$.032^{\mathrm{a}, \mathrm{d}}$

$.357^{\mathrm{c}}$

$.910^{c}$

$.167^{\mathrm{b}}$

$.767^{\mathrm{d}}$

$.004^{\mathrm{a}, \mathrm{c}}$

$.069^{c}$

$.031^{\mathrm{a}, \mathrm{c}}$

$.955^{\mathrm{c}}$ scores). Age, sex, and years of education were also added as independent variables in these models because of their expected influence on cognitive test scores, while the volumetric scaling factor was included to control for differences in head size between patients. For all analyses, 2-tailed $P$ values were used and $P<.05$ was considered significant.

Image-Based Analyses. Statistical maps were generated by using the Query, Design, Estimate, Contrast application in FreeSurfer. Briefly, Query, Design, Estimate, Contrast fits a generalized linear model at each surface vertex to explain the data from all subjects in the study. A surface-based Gaussian smoothing kernel of full width at half maximum of 10 $\mathrm{mm}$ was applied to the data before subsequent analyses. Using a generalized linear model, we compared cortical thickness variations between PD-MCI and PD-NCI, controlling for age, sex, and years of education. Correlations between regional cortical thickness and disease and cognitive measures were also modeled. The level of significance was set at $P<.05$ corrected for multiple comparison by using the false discovery rate. $^{40}$

\section{RESULTS}

\section{Subject Demographic and Clinical Characteristics}

The group comparisons of demographic and cognitive characteristics of the study cohort are shown in Tables 1 and 2, respectively. Within the PD group, 25 were classified as having PD-MCI; 65, as having PD-NCI, and none as having PD-dementia. The PD-MCI group was significantly older than PD-NCI group, while the PD-NCI group was more educated. Both groups were comparable in terms of duration of disease, Unified Parkinson's Disease Rating Scale motor scores, and Hoehn and Yahr staging. Comparisons of the various cardiovascular risk factors demonstrated a significantly higher prevalence of patients with PD-MCI having diabetes mellitus $(P=.004)$ and hyperlipidemia $(P=.031)$, compared with patients with PD-NCI.

\section{Cognitive Performance}

Patients with PD-MCI had significantly lower scores on global cognition compared with those with PD-NCI (Mini-Mental State Examination, $P=.016$; Montreal Cognitive Assessment, $P<.018$; Global Index, $P=.008$ ) after correcting for age, sex, and years of education. Comparisons of individual neuropsychological tests are shown in Table 2. With the exception of visuospatial ability and episodic memory domains, the PD-MCI group performed significantly poorer in executive functioning, attention and working memory, and language abilities (all, $P \leq .023$ ).

\section{SDGM Comparisons}

Group comparisons of mean volumes for all SDGM structures are shown in Table 3. ANCOVA analyses, controlling for age, sex, education, and head size revealed significant reductions in volumes of the thalamus $(P=.03)$ and nucleus accumbens $(P=.04)$ of patients with PD-MCI compared with PD-NCI. 
Table 2: Comparison of neuropsychological measures between patients with Parkinson disease with and without mild cognitive impairment

\begin{tabular}{|c|c|c|c|}
\hline Neuropsychological Measures & $\begin{array}{c}\text { PD-NCI } \\
\text { (Mean) (SD) }\end{array}$ & $\begin{array}{c}\text { PD-MCI } \\
\text { (Mean) (SD) }\end{array}$ & $\begin{array}{c}\text { Adjusted } \\
P \text { Value }\end{array}$ \\
\hline \multicolumn{4}{|l|}{ Global cognition (mean) (SD) } \\
\hline MMSE & $28.4(1.6)$ & $26.7(2.6)$ & $.016^{\mathrm{a}, \mathrm{b}}$ \\
\hline MOCA & $27.0(2.9)$ & $24.5(2.4)$ & $.018^{\mathrm{a}, \mathrm{b}}$ \\
\hline ADASI1 & $6.8(4.2)$ & $9.8(3.5)$ & $.071^{\mathrm{a}, \mathrm{b}}$ \\
\hline Global Index & $0.1(0.5)$ & $-0.3(0.3)$ & $.008^{\mathrm{a}, \mathrm{b}}$ \\
\hline \multicolumn{4}{|l|}{ Cognitive tests (mean) (SD) } \\
\hline Clock drawing & $9.31(1.25)$ & $8.48(1.47)$ & .126 \\
\hline Frontal Assessment Battery & 16.69 (1.38) & $15.28(2.09)$ & .027 \\
\hline Digit Span & $17.42(3.50)$ & $15.04(2.24)$ & .015 \\
\hline Digit Cancellation & $19.86(6.54)$ & $15.08(6.45)$ & .063 \\
\hline Color Trail 1 & $80.48(37.59)$ & $137.24(82.08)$ & .008 \\
\hline Color Trail 2 & $138.94(60.75)$ & 229.58 (103.31) & .002 \\
\hline Maze & $18.63(10.59)$ & $30.80(17.83)$ & .005 \\
\hline Maze errors & $0.06(0.30)$ & $0.04(0.20)$ & .942 \\
\hline ADAS: Immediate and Delayed Recall & $5.08(2.69)$ & $7.04(3.28)$ & .090 \\
\hline ADAS: Recognition & $1.55(2.22)$ & $2.00(2.50)$ & .644 \\
\hline ADAS: Language & $1.15(1.37)$ & $2.00(1.71)$ & .168 \\
\hline Fruit Fluency & $14.11(3.29)$ & $11.24(2.80)$ & .029 \\
\hline \multicolumn{4}{|l|}{ Cognitive domains (mean) (SD) } \\
\hline Executive function & $0.4(1.5)$ & $-1.0(2.0)$ & $.023^{a, b}$ \\
\hline Attention/working memory & $0.8(1.7)$ & $-2.0(2.4)$ & $<.000^{\mathrm{a}, \mathrm{b}}$ \\
\hline Visuospatial & $0.0(1.8)$ & $-0.1(1.4)$ & $.919^{d}$ \\
\hline Episodic memory & $0.3(1.6)$ & $-0.6(1.5)$ & $.193^{\mathrm{b}}$ \\
\hline Language & $0.4(1.4)$ & $-1.0(1.5)$ & $.018^{\mathrm{a}, \mathrm{b}}$ \\
\hline
\end{tabular}

Note:-MMSE indicates Mini-Mental State Examination; MOCA, Montreal Cognitive Assessment; ADASI1, Alzheimer Disease Assessment Scale-Cognitive.

a Significant differences at $P<.05$ level.

${ }^{b}$ Analysis of covariance test, corrected for age, sex, and education.

Table 3: Volumetric comparisons between patients with Parkinson disease with and without mild cognitive impairment

\begin{tabular}{lccc}
\multicolumn{1}{c}{ SDGM Structures $^{\mathrm{a}}$} & $\begin{array}{c}\text { PD-NCI } \\
\text { (Mean) (SD) }\end{array}$ & $\begin{array}{c}\text { PD-MCI } \\
\text { (Mean) (SD) }\end{array}$ & $\begin{array}{c}\text { Adjusted } \\
\boldsymbol{P}^{\text {P Value }}\end{array}$ \\
\hline Amygdala & $2.13(0.51)$ & $2.23(0.36)$ & $.220^{\mathrm{b}}$ \\
Hippocampus & $7.65(0.97)$ & $7.18(0.79)$ & $.198^{\mathrm{b}}$ \\
Nucleus accumbens & $0.83(0.22)$ & $0.64(0.19)$ & $.044^{\mathrm{b}}$ \\
Caudate nucleus & $6.37(0.76)$ & $5.96(0.76)$ & $.327^{\mathrm{b}}$ \\
Putamen & $9.51(1.41)$ & $8.62(1.17)$ & $.058^{\mathrm{b}}$ \\
Pallidum & $3.72(0.66)$ & $3.60(0.84)$ & $.876^{\mathrm{b}}$ \\
Thalamus & $13.94(1.65)$ & $12.71(1.09)$ & $.025^{\mathrm{b}}$ \\
Normalized gray matter & $688.76(34.86)$ & $661.92(28.24)$ & $.035^{\mathrm{b}}$ \\
Normalized white matter & $654.43(41.49)$ & $633.10(46.04)$ & $.618^{\mathrm{b}}$ \\
\hline
\end{tabular}

${ }^{a}$ All volumes are reported in milliliters. Significant difference is at the $P<.05$ level.

${ }^{\mathrm{b}}$ Analysis of variance adjusting for age, sex, education, and head size.

Table 4: Mean global and hemispheric cortical thickness between patients with Parkinson disease with and without mild cognitive impairment

\begin{tabular}{|c|c|c|c|}
\hline Cortical Thickness $^{a}$ & $\begin{array}{c}\text { PD-NCI } \\
\text { (Mean) (SD) }\end{array}$ & $\begin{array}{c}\text { PD-MCI } \\
\text { (Mean) (SD) }\end{array}$ & $\begin{array}{l}\text { Adjusted } \\
P \text { Value }\end{array}$ \\
\hline Left hemisphere & $2.20(0.10)$ & $2.13(0.11)$ & $.095^{b}$ \\
\hline Right hemisphere & $2.19(0.10)$ & $2.13(0.10)$ & $.264^{b}$ \\
\hline Global mean cortical thickness & $4.40(0.19)$ & $4.26(0.21)$ & $.151^{b}$ \\
\hline
\end{tabular}

${ }^{a}$ Cortical thickness is expressed in millimeters. Significant difference is at the $P<.05$ level.

${ }^{\mathrm{b}}$ Analysis of variance adjusting for age, sex, education

\section{Vertex-Wise Shape Comparisons of SDGM Structures}

Vertex analysis did not reveal any significant differences in the shapes of SDGM structures between PD-MCI and PD-NCI.

\section{Cortical Thickness Comparisons}

Global mean and hemispheric cortical thickness values are shown in Table 4. Vertex-wise group comparisons of regional cortical multiple comparisons.

\section{DISCUSSION}

thickness showed no significant differences between PD-MCI and PD-NCI in any cortical area.

\section{Association between SDGM Structures and Cognitive Impairment}

$\beta$ regression coefficients of the correlations between volumes of SDGM structures and the main cognitive tests scores are shown in the On-line Table. The total volume of the nucleus accumbens was significantly correlated with a range of cognitive variables, including overall scores of attention/working memory $\left(\beta=0.28, r^{2}=0.32, P=.02\right)$ and language $\left(\beta=0.25, r^{2}=0.29, P=.04\right)$ (Fig $2)$. Furthermore, there was a trend toward the associations between nucleus accumbens and global cognition $(\beta=$ $\left.0.23, r^{2}=0.2292, P=.06\right)$ and executive function $\left(\beta=0.23, r^{2}=0.2575, P=\right.$ .06). Putaminal volume was correlated with overall scores of attention/working memory $\left(\beta=0.31, r^{2}=0.34, P=.005\right)$. These correlations did not survive correction for multiple comparisons.

\section{Association between SDGM Structures and Disease Characteristics}

$\beta$ regression coefficients of the correlations between volumes of SDGM structures and the indices of disease characteristics (Unified Parkinson's Disease Rating Scale and disease duration) are shown in the On-line Table. Putamen volume was correlated with the Unified Parkinson's Disease Rating Scale $\left(\beta=-0.33, r^{2}=\right.$ $0.12, P=.009)$. This correlation did not survive correction for

At present, the pathophysiologic relationship between neurodegeneration processes and cognitive dysfunction in PD remains unclear, and ongoing investigations to identify structural biomarkers of cognitive impairment in patients with $\mathrm{PD}$ without dementia have yielded inconclusive results. Varying degrees of atrophy have been reported in patients with $\mathrm{PD}$ without dementia, ${ }^{41-43}$ with mixed evidence of an association between atrophy and neuropsychological measures. ${ }^{44}$ Within a cohort of 90 patients with mild PD without dementia, we investigated SDGM volumes and cortical thickness and examined their associations with cognitive functioning and disease severity. To date, only a few studies have directly examined the patterns of GM atrophy in well-delineated cognitive subgroups of PD. PD-MCI exhibited significantly reduced total volumes in a number of SDGM regions, including the thalamus and nucleus accumbens, and there was a trend toward the putamen, relative to PD-NCI.

These volumetric reductions suggest that a pattern of subcortical atrophy can be detected at an early stage of cognitive decline 
in mild stages of PD. The finding of thalamic atrophy in PD-MCI is novel, and warrants further consideration. Traditionally, the thalamus has been conceptualized as a relay center, involved in both sensory and motor functions. ${ }^{45}$ It is increasingly recognized as an important site for neuropathologic inclusions, including Lewy bodies in patients with PD. The relationship between thalamic degeneration and cognitive impairment has also been investigated in previous studies. Volumetric loss in the thalamus has been proposed as a predictor of dementia, ${ }^{46}$ while studies have demonstrated thalamic atrophy in PD with dementia compared with healthy individuals. ${ }^{14,16}$ Previous studies have also demonstrated the relation of thalamic atrophy to cognitive performance in other neurologic disorders, including Alzheimer disease, ${ }^{47}$ Huntington disease, ${ }^{48}$ and multiple sclerosis. ${ }^{49}$ Considered in light of those findings, the volumetric reduction of the thalamus observed in our PD-MCI group may reflect an intermediary stage of cognitive dysfunction. Of note, we did not find any significant alteration of the shapes of SDGM structures in PD-MCI relative to PD-NCI. Further studies are needed to examine the clinical significance of shape alterations in SDGM structures and their utility as potential biomarkers of cognitive impairment and dementia in PD.

Additionally, a number of associations between the nucleus accumbens and putamen and cognitive test scores were also found. While the results were controlled for age, sex, years of education, and head size, they were not corrected for multiple comparisons. Performance on attention and working memory was associated with reduced volumes of the nucleus accumbens and putamen. Additionally, the nucleus accumbens was also significantly correlated with performance in the language domain. A possible explanation for our findings might be offered by previous evidence linking the nucleus accumbens to memory and learning processes. $^{50}$ Furthermore, a previous population-based study demonstrated that accumbens volume is predictive of cognitive decline in the elderly. ${ }^{51}$ At this time, very little is known about the putaminal role in cognition. Despite the traditional role of the putamen in motor functions, the finding of an association between reduced putaminal volumes and cognitive scores on the attention and working memory domain in the present study is consistent with a previous report that demonstrated a signification association between putaminal 6-[ $\left[{ }^{18} \mathrm{~F}\right]$-fluoro-L-dopa uptake and measures for executive functioning, memory, and fluency in a group of 28 patients with PD without dementia. ${ }^{52}$

At present, the literature concerning the role of cortical thinning in cognitive deterioration in PD remains inconclusive. We did not find significant thinning of the cortex in PD-MCI compared with PD-NCI in any region. A previous study found significant associations between the Mini-Mental State Examination and cortical thickness in their PD cohort. ${ }^{53}$ In addition, they found significant cortical thinning in patients with moderate PD without dementia compared with healthy controls and in PD with dementia compared with moderate PD without dementia. Considered together, the absence of cortical thinning in our PD-MCI group might be attributed to the finer distinction of cognitive statuses in our PD cohort.

The strengths of this study include the use of the MDS Task Force diagnostic criteria and a comprehensive neuropsychologi- cal evaluation to characterize PD-MCI. However, we acknowledge the possible involvement of executive functions in the interpretation of the fluency test findings that was used to characterize language in our cohort. In-painted T1 images also improved the quality of the subcortical segmentation due to the known effect of tissue misclassification due to lesion-induced T1 hypointensities in the WM. Preprocessing of images to correct for such pathology is now highly recommended.

Our study was limited by the absence of patients with PD with established dementia and healthy controls for comparison. This constraint prevented us from exploring the associations between SDGM volumes and cognitive dysfunction across a broader range of cognitive stages in PD. As such, these findings need to be confirmed in larger prospective studies with an additional group of well-matched healthy controls. In this regard, a recent study has also demonstrated subcortical atrophy in PD without dementia compared with healthy controls, particularly in the putamen, nucleus accumbens, and hippocampus. ${ }^{54}$ In addition, the present findings must be interpreted by taking into account the lack of correction for multiple comparisons and that the sample size between PD-NCI $(n=65)$ and PD-MCI $(n=25)$ was imbalanced. Thus, to check against a potential violation of the assumption of equal variances across groups in ANOVA, we performed the Bartlett test to ensure homoscedasticity. Another limitation of the study could be related to performance of testing during the "on" medication state. Moreover, we did not have volumetric data on the substantia nigra, an area with extensive projections to limbic and cortical regions. In fact, an earlier study found a significant correlation between the severity of dementia in patients with PD and neuronal loss in the medial part of the substantia nigra. ${ }^{55}$ Finally, we aim to extend this study by incorporating a longitudinal design that will allow us to elucidate the trajectory of brain atrophy in PD-MCI and examine its potential involvement in progression to $\mathrm{PD}$ dementia.

\section{CONCLUSIONS}

Due to the growing recognition of PD-MCI as a clinically significant condition in $\mathrm{PD}$, our findings warrant the continued concerted effort to validate biomarkers of neurodegeneration associated with MCI. The early delineation of PD-MCI from PD-NCI will help elucidate the processes underlying cognitive decline in $\mathrm{PD}$, while longitudinal research can investigate the contributions of SDGM structures to cognitive dysfunction as the disease progresses.

Disclosures: Michael G. Dwyer-UNRELATED: Consultancy: Claret Medical, EMD Serono, Comments: served on scientific advisory board for EMD Serono and has done image analysis consulting work for Claret Medical. Robert ZivadinovUNRELATED: Consultancy: Biogen Idec, EMD Serono, Teva Pharmaceuticals, Genzyme-Sanofi, Novartis, Claret Medical; Grants/Grants Pending: Biogen Idec, ${ }^{*}$ EMD Serono, ${ }^{*}$ Teva Pharmaceuticals, ${ }^{*}$ Novartis, ${ }^{\star}$ Genzyme-Sanofi, ${ }^{*}$ Claret Medical*; Payment for Lectures (including service on Speakers Bureaus): Biogen Idec, EMD Serono, Teva Pharmaceuticals, Genzyme-Sanofi, Novartis. Nagaendran KandiahRELATED: Grant: National Medical Research Council of Singapore*; UNRELATED: Grants/Grants Pending: National Medical Research Council of Singapore, ${ }^{*}$ Biomedical Research Council of Singapore, ${ }^{*}$ Singhealth Foundation, ${ }^{*}$ Media Development Authority of Singapore*; Travel/Accommodations/Meeting Expenses Unrelated to Activities Listed: Novartis, ${ }^{*}$ Eisai, ${ }^{*}$ Lundbeck*; Other: N.K. has received an honorarium and Continuing Medical Education sponsorship from Lundbeck.* *Money paid to the institution. 


\section{REFERENCES}

1. Muslimovic D, Post B, Speelman JD, et al. Cognitive profile of patients with newly diagnosed Parkinson disease. Neurology 2005;65:1239-45

2. Sollinger AB, Goldstein FC, Lah JJ, et al. Mild cognitive impairment in Parkinson's disease: subtypes and motor characteristics. Parkinsonism Relat Disord 2010;16:177-80

3. Poletti M, Emre M, Bonuccelli U. Mild cognitive impairment and cognitive reserve in Parkinson's disease. Parkinsonism Relat Disord 2011;17:579-86

4. Kandiah N, Narasimhalu K, Lau PN, et al. Cognitive decline in early Parkinson's disease. Mov Disord 2009;24:605-08

5. Caviness JN, Driver-Dunckley E, Connor DJ, et al. Defining mild cognitive impairment in Parkinson's disease. Mov Disord 2007; 22:1272-77

6. Litvan I, Aarsland D, Adler CH, et al. MDS task force on mild cognitive impairment in Parkinson's disease: critical review of PD-MCI. Mov Disord 2011;26:1814-24

7. Bronnick K, Ehrt U, Emre M, et al. Attentional deficits affect activities of daily living in dementia-associated with Parkinson's disease. J Neurol Neurosurg Psychiatry 2006;77:1136-42

8. Aarsland D, Bronnick K, Ehrt U, et al. Neuropsychiatric symptoms in patients with Parkinson's disease and dementia: frequency, profile and associated care giver stress. J Neurol Neurosurg Psychiatry 20062007;78:36-42

9. Levy G, Tang MX, Louis ED, et al. The association of incident dementia with mortality in PD. Neurology 2002;59:1708-13

10. Owen AM. Cognitive dysfunction in Parkinson's disease: the role of frontostriatal circuitry. Neuroscientist 2004;10:525-37

11. Calabresi P, Picconi B, Parnetti L, et al. A convergent model for cognitive dysfunctions in Parkinson's disease: the critical dopamineacetylcholine synaptic balance. Lancet Neurol 2006;5:974-83

12. Pan PL, Shi HC, Zhong JG, et al. Gray matter atrophy in Parkinson's disease with dementia: evidence from meta-analysis of voxel-based morphometry studies. Neurol Sci 2013;34:613-19

13. Almeida OP, Burton EJ, McKeith I, et al. MRI study of caudate nucleus volume in Parkinson's disease with and without dementia with Lewy bodies and Alzheimer's disease. Dement Geriatr Cogn Disord 2003;16:57-63

14. Burton EJ, McKeith IG, Burn DJ, et al. Cerebral atrophy in Parkinson's disease with and without dementia: a comparison with Alzheimer's disease, dementia with Lewy bodies and controls. Brain 2004;127:791-800

15. Junqué C, Ramirez-Ruiz B, Tolosa E, et al. Amygdalar and hippocampal MRI volumetric reductions in Parkinson's disease with dementia. Mov Disord 2005;20:540-44

16. Summerfield C, Junque C, Tolosa E, et al. Structural brain changes in Parkinson disease with dementia: a voxel-based morphometry study. Arch Neurol 2005;62:281-85

17. Bouchard TP, Malykhin N, Martin WR, et al. Age and dementiaassociated atrophy predominates in the hippocampal head and amygdala in Parkinson's disease. Neurobiol Aging 2008;29:1027-39

18. Nishio Y, Hirayama K, Takeda A, et al. Corticolimbic gray matter loss in Parkinson's disease without dementia. Eur J Neurol 2010;17:1090-97

19. Tessa C, Giannelli M, Della Nave R, et al. A whole-brain analysis in de novo Parkinson disease. AJNR Am J Neuroradiol 2008;29:674-80

20. Pereira JB, Ibarretxe-Bilbao N, Marti MJ, et al. Assessment of cortical degeneration in patients with Parkinson's disease by voxel-based morphometry, cortical folding, and cortical thickness. Hum Brain Mapping 2012;33:2521-34

21. Tinaz S, Courtney MG, Stern CE. Focal cortical and subcortical atrophy in early Parkinson's disease. Mov Disord 2011;26:436-41

22. Ibarretxe-Bilbao N, Junque C, Segura B, et al. Progression of cortical thinning in early Parkinson's disease. Mov Disord 2012;27:1746-53

23. Patenaude B, Smith SM, Kennedy DN, et al. A Bayesian model of shape and appearance for subcortical brain segmentation. Neuroimage 2011;56:907-22
24. Gelb DJ, Oliver E, Gilman S. Diagnostic criteria for Parkinson disease. Arch Neurol 1999;56:33-39

25. Movement Disorder Society Task Force on Rating Scales for Parkinson's Disease. The Unified Parkinson's Disease Rating Scale (UPDRS): status and recommendations. Mov Disord 2003;18: $738-50$

26. Hoehn MM, Yahr MD. Parkinsonism: onset, progression, and mortality. Neurology 1967;17:427-42

27. Tomlinson CL, Stowe R, Patel S, et al. Systematic review of levodopa dose equivalency reporting in Parkinson's disease. Mov Disord 2010;25:2649-53

28. Folstein MF, Robins LN, Helzer JE. The Mini-Mental State Examination. Arch Gen Psychiatry 1983;40:812

29. Dalrymple-Alford JC, MacAskill MR, Nakas CT, et al. The MoCA well-suited screen for cognitive impairment in Parkinson disease. Neurology 2010;75:1717-25

30. Litvan I, Goldman JG, Tröster AI, et al. Diagnostic criteria for mild cognitive impairment in Parkinson's disease: Movement Disorder Society Task Force guidelines. Mov Disord 2012;27:349-56

31. Rosen WG, Mohs RC, and Davis KL. A new rating scale for Alzheimer's disease. Am J Psychiatry 1984;141:1356-64

32. Dubois B, Slachevsky A, Litvan I, et al. The FAB: a frontal assessment battery at bedside. Neurology 2000;55:1621-26

33. Sunderland T, Hill JL, Mellow AM, et al. Clock drawing in Alzheimer's disease: a novel measure of dementia severity. J Am Geriatr Soc 1989;37:725-29

34. Jenkinson M, Beckmann CF, Behrens TE, et al. FSL. Neuroimage 2012;62:782-90

35. Gelineau-Morel R, Tomassini V, Jenkinson M, et al. The effect of hypointense white matter lesions on automated gray matter segmentation in multiple sclerosis. Hum Brain Mapping 2012;33: 2802-14

36. Zivadinov R, Heininen-Brown M, Schirda CV, et al. Abnormal subcortical deep-gray matter susceptibility-weighted imaging filtered phase measurements in patients with multiple sclerosis: a case-control study. Neuroimage 2012;59:331-39

37. Batista S, Zivadinov R, Hoogs $M$, et al. Basal ganglia, thalamus and neocortical atrophy predicting slowed cognitive processing in multiple sclerosis. J Neurol 2012;259:139-46

38. Hughes EJ, Bond J, Svrckova P, et al. Regional changes in thalamic shape and volume with increasing age. Neuroimage 2012;63: 1134-42

39. Fischl B. FreeSurfer. Neuroimage 2012;62:774-81

40. Genovese CR, Lazar NA, and Nichols T. Thresholding of statistical maps in functional neuroimaging using the false discovery rate. Neuroimage 2002;15:870-78

41. Lyoo CH, Ryu YH, and Lee MS. Topographical distribution of cerebral cortical thinning in patients with mild Parkinson's disease without dementia. Mov Disord 2010;25:496-99

42. Melzer TR, Watts R, MacAskill MR, et al. Grey matter atrophy in cognitively impaired Parkinson's disease. J Neurol Neurosurg Psychiatry 2012;83:188-94

43. Song SK, Lee JE, Park HJ, et al. The pattern of cortical atrophy in patients with Parkinson's disease according to cognitive status. Mov Disord 2011;26:289-96

44. Dalaker TO, Zivadinov R, Larsen JP, et al. Gray matter correlations of cognition in incident Parkinson's disease. Mov Disord 2010; 25:629-33

45. Herrero MT, Barcia C, Navarro JM. Functional anatomy of thalamus and basal ganglia. Childs Nerv Syst 2002;18:386-404

46. de la Monte SM, Wells SE, Hedley-Whyte T, et al. Neuropathological distinction between Parkinson's dementia and Parkinson's plus Alzheimer's disease. Ann Neurol 1989;26:309-20

47. de Jong LW, van der Hiele K, Veer IM, et al. Strongly reduced volumes of putamen and thalamus in Alzheimer's disease: an MRI study. Brain 2008;131:3277-85

48. Kassubek J, Juengling FD, Ecker D, et al. Thalamic atrophy in Hun- 
tington's disease co-varies with cognitive performance: a morphometric MRI analysis. Cereb Cortex 2005;15:846-53

49. Benedict, RH, Hulst HE, Bergsland N, et al. Clinical significance of atrophy and white matter mean diffusivity within the thalamus of multiple sclerosis patients. Mult Scler 2013;19:1478-84

50. Goldenberg G, Schuri U, Gromminger O, et al. Basal forebrain amnesia: does the nucleus accumbens contribute to human memory? J Neurol Neurosurg Psychiatry 1999;67:163-68

51. de Jong LW, Wang Y, White LR, et al. Ventral striatal volume is associated with cognitive decline in older people: a population based MR-study. Neurobiol Aging 2012;33:424.e1-10
52. van Beilen M, Leenders KL. Putamen FDOPA uptake and its relationship to cognitive functioning in PD. J Neurol Sci 2006;248:68-71

53. Zarei M, Ibarretxe-Bilbao N, Compta $\mathrm{Y}$, et al. Cortical thinning is associated with disease stages and dementia in Parkinson's disease. J Neurol Neurosurg Psychiatry 2013;84:875-81

54. Lee HM, Kwo, KY, Kim MJ, et al. Subcortical grey matter changes in untreated, early stage Parkinson's disease without dementia. Parkinsonism Relat Disord 2014;20:622-26

55. Rinne JO, Mlic JR, Paljärvi L, et al. Dementia in Parkinson's disease is related to neuronal loss in the medial substantia nigra. Ann Neurol 1989;26:47-50 